\title{
Kernos
}

Revue internationale et pluridisciplinaire de religion grecque antique

$28 \mid 2015$

Varia

\section{The Oxford Handbook of Animals in Classical Thought and Life}

\section{Zoé Pitz}

\section{(2) OpenEdition \\ Journals}

\section{Édition électronique}

URL : http://journals.openedition.org/kernos/2362

DOI : 10.4000/kernos.2362

ISSN : 2034-7871

\section{Éditeur}

Centre international d'étude de la religion grecque antique

\section{Édition imprimée}

Date de publication : 1 octobre 2015

Pagination : 292-294

ISBN : 978-2-87562-055-2

ISSN : 0776-3824

\section{Référence électronique}

Zoé Pitz, "The Oxford Handbook of Animals in Classical Thought and Life », Kernos [En ligne], 28 |

2015, mis en ligne le 01 octobre 2015, consulté le 24 septembre 2020. URL : http://

journals.openedition.org/kernos/2362 ; DOI : https://doi.org/10.4000/kernos.2362

Ce document a été généré automatiquement le 24 septembre 2020

Kernos 


\title{
The Oxford Handbook of Animals in Classical Thought and Life
}

\author{
Zoé Pitz
}

\section{RÉFÉRENCE}

CAMPBELL Gordon L. (éd.), The Oxford Handbook of Animals in Classical Thought and Life, Oxford, Oxford University Press, 2014.1 vol. $18 \times 25,5 \mathrm{~cm}$. ix+633 p. ISBN :

978-0-19-958942-5.

1 Cet ouvrage, qui rassemble les études de trente-trois chercheurs en autant de chapitres, est le premier manuel intégral sur les animaux dans le monde ancien. Il aborde de nombreux thèmes en lien avec l'animal dans l'Antiquité, tant concrets - la domestication, l'élevage, la pêche, l'usage des animaux dans les triomphes romains qu'abstraits - la communication animale, l'origine des espèces, le végétarisme, les connaissances zoologiques dans l'Antiquité ${ }^{1}$. Tous les chapitres contiennent une introduction et un aperçu de la problématique, et la plupart d'entre eux proposent également des pistes de lecture, destinées à ceux qui souhaiteraient approfondir leur connaissance des différents thèmes. Les chapitres 18 à 23 , qui portent sur des questions religieuses, seront présentés ici.

Quatre thèmes sont étudiés successivement par D. Ogden dans son chapitre «Animal Magic» (p. 294-309) : la transformation des humains en animaux, l'exploitation des animaux - et en particulier des parties animales - à des fins magiques, le déploiement de la magie contre les animaux et le déploiement de la magie par les animaux contre les hommes. Le plus long développement, initié par un exemple de l'Histoire Naturelle de Pline - l'emploi des parties de la hyène dans les prescriptions des mages -, est consacré au lien entre le choix des parties animales, leur mode d'utilisation et le résultat magique escompté. Dans le cadre de ce développement, l'auteur s'interroge notamment sur l'origine $\mathrm{du}$ pouvoir magique: celui-ci réside-t-il dans les parties animales elles-mêmes ou se déploie-t-il suite à leur manipulation rituelle? 
3 Dans «Animals and Divination » (p. 310-323), P. Struck s'intéresse à la place occupée par les animaux dans les différentes techniques de divination utilisées dans l'Antiquité. L'auteur étudie dans un premier temps les comportements instinctifs des animaux, que les Grecs et les Romains considéraient comme des signes divins, en insistant particulièrement sur l'examen du comportement des oiseaux. Il aborde ensuite la divination par les entrailles des animaux sacrifiés. Pour terminer, P. Struck propose une réflexion sur les théories divinatoires anciennes, où il expose de manière relativement détaillée les conceptions de Platon, d'Aristote et des Stoïciens.

4 Le chapitre « Animal Sacrifice in Antiquity » (p. 324-354), rédigé par G. Ekroth, est l'un des plus longs de l'ouvrage. Il se distingue de bon nombre d'études antérieures sur le sacrifice, notamment parce que l'auteur y présente quelques hypothèses assez novatrices, fondées sur les résultats de fouilles archéozoologiques. Après avoir rappelé quelques généralités sur le déroulement des différents rituels sacrificiels pratiqués dans l'Antiquité, G. Ekroth aborde la question des critères qui président au choix d'un animal sacrificiel. Elle livre ensuite les apports de l'analyse archéozoologique opérée dans les dépôts sacrificiels et s'interroge sur le statut à conférer aux restes d'animaux exotiques et sauvages mis au jour dans ces dépôts : ces animaux étaient-ils amenés vivants dans les sanctuaires afin d'y être sacrifiés et consommés, ou certaines parties la peau, les dents, les cormes ou les griffes - y étaient-elles apportées en tant qu'objets dédicatoires? L'auteur s'intéresse en outre à plusieurs espèces retrouvées dans les sanctuaires parmi les restes de repas, mais qui n'étaient vraisemblablement pas des victimes sacrificielles au même titre que les bœufs, les moutons ou les chèvres. Finalement, après une réflexion sur la consommation de viande dans l'Antiquité grécoromaine, G. Ekroth clôt son chapitre par un retour aux origines du sacrifice animal et un bref aperçu des critiques formulées à l'encontre de cette pratique rituelle.

Dans « Animals in Late Antiquity and Early Christianity » (p. 355-365), I.S. Gilhus étudie le sort réservé aux animaux lors du passage d'une culture païenne à une culture chrétienne dans le monde romain. Après s'être penchée brièvement sur la vision de l'animal dans les écrits chrétiens, l'auteur aborde la question de la réinterprétation chrétienne du sacrifice animal et de l'arène : la terminologie sacrificielle est transférée des animaux aux êtres humains - le Christ, les martyrs, les ascètes, etc. - et l'arène devient le lieu d'une lutte cosmologique entre Dieu et Satan, dont les animaux sont fréquemment les représentants. Vient ensuite une réflexion sur les animaux présents dans le désert: dans cet espace religieux créé par le christianisme, ceux-ci endossent également le rôle d'instruments $\mathrm{du}$ «bien» ou du «mal». Enfin, I.S. Gilhus se préoccupe de l'intérêt porté aux animaux par les auteurs chrétiens, ainsi que du rapport des Chrétiens à la viande - rejet de la viande sacrificielle et végétarisme.

6 Le chapitre "Part-Animal Gods» (p.366-383), proposé par E. Aston, traite de la représentation des dieux sous une forme hybride, à la fois thériomorphique et anthropomorphique. Dans un premier temps, l'auteur s'interroge sur deux questions essentielles posées par l'hybridisme : existe-t-il une "signification » des espèces, qui pousserait à choisir un animal plutôt qu'un autre comme composante de l'anatomie d'une divinité? De même, peut-on distinguer une logique à l'œuvre dans la combinaison des parties animales et humaines ? E. Aston envisage ensuite le rapport de l'hybridisme avec la monstruosité, ainsi que l'association entre l'hybridisme et le dieu Dionysos. Après une réflexion sur les divinités aquatiques, qui ont, comme Dionysos, 
tendance à changer de forme, l'auteur achève son chapitre par l'analyse des cultes des dieux hybrides.

7 Les métamorphoses en animaux sont étudiées par C. Thumiger dans « Metamorphosis : Human into Animals» (p.384-413). L'auteur propose tout d'abord d'opérer un classement entre les différents types de métamorphoses en animaux, notamment en fonction de la nature divine ou humaine des protagonistes. Elle considère ensuite deux modes de représentation de la métamorphose, tant dans la littérature que dans l'art figuratif : diachronique - le processus de transformation est placé au premier plan ou synchronique - l'accent est mis sur la coexistence des différentes natures de l'individu. Enfin, la plus grande partie du chapitre est consacrée à l'étude de la métamorphose à travers différents genres littéraires et auteurs anciens : la littérature épique, le théâtre, la littérature hellénistique, Ovide, Apulée, etc.

L'intérêt de cet ouvrage réside incontestablement dans la possibilité de lire chaque chapitre de manière indépendante ou d'envisager le livre comme un tout organisé sur le thème de l'animal dans l'Antiquité. Un autre point positif consiste en la présence d'un index des notions, très utile dans un volume d'une telle ampleur, rassemblant des sujets parfois si différents les uns des autres.

\section{NOTES}

1. Depuis la parution de l'ouvrage, l'auteur du chapitre « Zoological Knowledge in Ancient Greece and Rome » a créé un fichier PDF corrigeant plusieurs erreurs commises lors de la publication et annulant dès lors les versions imprimée et électronique éditées par Oxford University Press. Ce fichier PDF est disponible à l'adresse http://www.promethee.philo.ulg.ac.be/Zoologica/lbodson/ bibl/.

\section{AUTEURS}

\section{ZOÉ PITZ}

F.R.S.-FNRS - Université de Liège 